\title{
Immune characterization of long pentraxin 3 in pigs infected with influenza virus
}

\author{
Elisa Crisci ${ }^{a}$, Lorenzo Fraile ${ }^{\mathrm{a}, \mathrm{b}}$, Sonia Valentino ${ }^{\mathrm{c}}$, Laura Martínez-Guinó ${ }^{\mathrm{a}}$, \\ Barbara Bottazzi ${ }^{c}$, Alberto Mantovani ${ }^{\mathrm{c}, \mathrm{d}}$, Maria Montoya ${ }^{\mathrm{a}, \mathrm{e}, *}$ \\ ${ }^{a}$ Centre de Recerca en Sanitat Animal (CReSA), UAB-IRTA, Campus de la Universitat Autònoma de Barcelona, 08193 Bellaterra, Barcelona, \\ Spain \\ ${ }^{\mathrm{b}}$ Universitat de Lleida, Lleida, Spain \\ ${ }^{\mathrm{c}}$ Humanitas Clinical and Research Center, Rozzano, Italy \\ ${ }^{\mathrm{d}}$ Department of Translational Medicine, University of Milan, Rozzano, Italy \\ e Institut de Recerca i Tecnologia Agroalimentàries (IRTA), Barcelona, Spain
}

\section{A R T I C L E I N F O}

\section{Article history:}

Received 21 March 2013

Received in revised form 27 September 2013 Accepted 4 October 2013

\section{Keywords:}

PTX3

Pigs

DC

Influenza virus

\begin{abstract}
A B S T R A C T
Long pentraxin 3 (PTX3) is a conserved pattern-recognition secreted protein and a hostdefence-related component of the humoral innate immune system. The aim of the present study was to characterize swine PTX3 (SwPTX3) protein expression in influenza virus infected pigs. First, we performed in silico studies to evaluate the cross-reactivity of PTX3 human antibodies against SwPTX3. Secondly, we used in vitro analysis to detect SwPTX3 presence in swine bone marrow dendritic cells (SwBMDC) upon stimulation with different agents by Western blot and immunofluorescence. Finally, the levels of SwPTX3 were assessed in experimental infection of pigs with different strains of influenza virus. This is a novel study where the expression of SwPTX3 was evaluated in the context of a pathogen infection. The initial characterization of SwPTX3 in influenza virus infected pigs contributes to understand the role of PTX proteins in the immune response.
\end{abstract}

(c) 2013 Elsevier B.V. All rights reserved.

\section{Introduction}

The innate immune system constitutes the first line of defence against microorganisms and plays a primordial role in the activation and regulation of adaptive immunity. Components of the humoral arm include members of the complement cascade and soluble pattern recognition molecules (PRMs), such as collectins, ficolins and pentraxins (Bottazzi et al., 2010; Deban et al., 2011). Pentraxins constitute a superfamily of acute phase multifunctional proteins with multimeric organization that are phylogenetically conserved from arachnids to

\footnotetext{
* Corresponding author at: CReSA (Centro de Recerca en Sanitat Animal), Campus UAB, 08193 Bellaterra, Barcelona, Spain. Tel.: +34 935814562; fax: +34935814490.

E-mail address: maria.montoya@cresa.uab.es (M. Montoya).
}

mammals representing the prototypic component of humoral innate immunity (Bottazzi et al., 2009; Deban et al., 2011; Inforzato et al., 2011). Long pentraxin 3 (PTX3) is the prototype of this family and human PTX3 (HuPTX3) comprise a C-terminal pentraxin-like domain of 203 amino acids (aa) and 178 aa N-terminal domain (Bottazzi et al., 1997; Inforzato et al., 2006).

A variety of cell types express PTX3 upon exposure to inflammatory signals, such as cytokines (e.g. IL-1 $\beta$, TNF- $\alpha$ ), toll-like receptor (TLR) agonists, microbial moieties (e.g. LPS) or microorganisms (Bottazzi et al., 2009; Cieslik and Hrycek, 2012; Deban et al., 2011; Inforzato et al., 2011). Myeloid dendritic cells (DCs) are the major source of PTX3, although this molecule is also expressed by a number of other cell types (Bottazzi et al., 2010; Cieslik and Hrycek, 2012; Deban et al., 2011; Inforzato et al., 2011). Neutrophils are a reservoir of ready-to-use PTX3, released in minutes, whereas DCs and macrophages produce this molecule de 
novo in response to inflammatory signals (Bottazzi et al., 2009; Jaillon et al., 2007). The data indicate that PTX3 is involved in a variety of defence mechanisms, including resistance against some viral infections. Indeed, human and murine PTX3 bind influenza virus (IV) through interaction between viral haemagglutinin (HA) glycoprotein and the sialic acid residue present on PTX3. PTX3 inhibits virusinduced haemagglutination and viral neuraminidase (NA) activity and it neutralizes viral infectivity in vitro (Reading et al., 2008). This in vitro activity has been also observed in vivo in terms of lower mortality and lesser viral load when using an influenza model in mice (Reading et al., 2008). A recent study has shown that both seasonal H1N1 and pandemic H1N1 IVs were resistant to PTX3 (Job et al., 2010). In addition to the specificity of HA, viral NA plays a critical role in determining PTX3 sensitivity, as the sialic acid moiety on PTX3 must resist hydrolysis by NA for neutralization to occur. In spite of the fact that PTX3 plays an important role in host defence, its over-expression can contribute to airway injury (He et al., 2007; Ramery et al., 2010).

The aim of this study was to characterize for the first time in vitro and in vivo SwPTX3 expression upon infection using human cross-reactive antibodies. This preliminary study will pave the way to understanding SwPTX3 function in pig innate immunity.

\section{Materials and methods}

\subsection{PTX3 and anti-PTX3 antibodies}

Recombinant human and murine PTX3 were purified under endotoxin-free conditions by immunoaffinity from the supernatants of stably transfected $\mathrm{CHO}$ cells, as previously described (Bottazzi et al., 1997).

Polyclonal rabbit antiserum was obtained by immunization with purified recombinant human PTX3. Specific IgG anti human PTX3 were subsequently purified by immunoaffinity on Cyanogen Bromide (CNBr)-activated Sepharose column conjugated with recombinant human PTX3 (CNBr-activated Sepharose 4 fast flow GE Healthcare, Milan, Italy) (Peri et al., 2000). Rat monoclonal antibodies MNB4 and 16B5 were raised against human PTX3 and shown to recognize respectively epitope (87-99) in the Nterminal domain and epitope (306-312) in the C-terminal domain (Camozzi et al., 2006). Biotinylated antibodies were obtained following incubation with N-hydroxysuccinimidobiotin (Biotin-NHS, Biospa, Milan, Italy). Briefly, purified antibodies $(1 \mathrm{mg} / \mathrm{ml})$ in $0.1 \mathrm{M}$ carbonate buffer $(\mathrm{pH} 8.5)$ were incubated $4 \mathrm{~h}$ at room temperature with Biotin-NHS ( $1 \mathrm{mg} / \mathrm{ml}$ in dimethyl sulfoxide) at the ratio 0.12:1 (v/v). After incubation antibodies were extensively dialyzed against PBS (Bottazzi et al., 1997).

\subsection{In silico study}

PTX3 sequences from human [GenBank accession number: NP_002843.2], murine [GenBank accession number: NP_033013.3] and swine [GenBank accession number: NP_001231712.1] were retrieved from the National Centre for Biotechnology Information (NCBI) database. Sequences were subjected to blast analysis [http://
www.ncbi.nlm.nih.gov/BLAST] and to alignment with Clustal W. (Higgins et al., 1996). Amino acids recognized by MNB4 and 16B5 antibodies in the human PTX3 sequence were compared to the swine PTX3 sequence. NetNglyc server was used to predict $\mathrm{N}$-glycosilation sites [http://www.cbs.dtu.dk/services/NetNGlyc/].

\subsection{Swine bone marrow derived dendritic cell generation}

Bone marrow hematopoietic cells were obtained from femurs of healthy Large white X Landrace pigs of eight weeks of age. Swine bone marrow dendritic cells (SwBMDCs) were generated in an eight days protocol as previously described by (Crisci et al., 2012; Mussa et al., 2011). Eight-day culture of immature SwBMDCs were used for infection or stimulation.

\subsection{Viruses and stimuli}

The novel human virus of swine origin, A/Catalonia/63/ 2009 (H1N1) IV (GenBank accession numbers GQ464405GQ464411 and GQ168897) was isolated in 2009 from a patient at the Hospital Clinic, Barcelona, Spain, and was propagated in the allantoic cavities of 11 day-old embryonated chicken eggs originating from a commercial specific-pathogen-free (SPF) flock (GDdeventer). Swine influenza virus (SwIV) H3N2 (A/Swine/Spain/SF32071/ 2007) and H1N2 (A/Swine/Spain/SF12091/2007) were isolated from farms in Catalonia. All influenza viruses were grown and titrated in MDCK cells, and virus titre was calculated by the Reed and Muench method (Reed and Muench, 1938).

For in vitro studies, SwBMDCs were infected with 1 multiplicity of infection (moi=1) with H3N2 SwIV (A/ Swine/Spain/SF32071/2007) as previously described (Mussa et al., 2011). In addition, SwBMDCs were stimulated with $10 \mu \mathrm{g} / \mathrm{ml}$ of LPS (Sigma-Aldrich) or $50 \mu \mathrm{g} / \mathrm{ml}$ of virus-like particles belonging to rabbit haemorrhagic disease calicivirus (RHDV-VLPs) (Crisci et al., 2012) for $8 \mathrm{~h}$ or overnight $(\mathrm{ON})$. Uninfected and unstimulated SwBMDCs were used as negative control.

\subsection{Western blot analysis}

SwBMDCs were infected overnight with IV, stimulated with LPS or with RHDV-VLPs. After incubation, cells were washed with $1 \times$ PBS and lysed with lysis buffer containing $50 \mathrm{mM}$ Tris-HCL, pH 8, $150 \mathrm{mM} \mathrm{NaCl}, 2$ mM EDTA, 10\% Triton X-100 and a protease inhibitor cocktail (SigmaAldrich) at $4{ }^{\circ} \mathrm{C}$. After centrifugation at $12,000 \times \mathrm{g}$ for $15 \mathrm{~min}$ at $4{ }^{\circ} \mathrm{C}$, supernatant (lysate) was collected.

Each lysate was generated from $3 \times 10^{6}$ total cells/ sample with an average of total protein concentration around $1.7 \mathrm{mg} / \mathrm{ml}$. Fifthteen microlitres of cell lysate (total volume $0.5 \mathrm{ml}$ ) were subjected to denaturing SDSPAGE (NuPAGE ${ }^{\circledR}$ Novex 4-12\% Bis-Tris gel; Invitrogen Corporation) followed by Western Blot analysis. Gels were electroblotted to a Hybond ECL ${ }^{\mathrm{TM}}$ nitrocellulose membrane (GE Healthcare) followed by blocking in 5\% skim milk. After incubation of membranes with primary antibody MNBA or 16B5 (1:10,000 dilution) at room 
temperature (RT) for $1 \mathrm{~h}$, three washes with tris-buffered saline $+0.1 \%$ Tween 20 (TBS-Tween) $10 \mathrm{~min}$ each followed. Anti-rat secondary antibody HRP conjugate (Invitrogen; 1:100,000 dilution) was added and membrane incubated at RT for $1 \mathrm{~h}$ followed by three washes. Proteins were revealed with ECL Advance ${ }^{\mathrm{TM}}$ Western Blotting Detection Kit (GE Healthcare) as described by the manufacturer. RPMI 1640 complete medium (LONZA) with $10 \%$ of FCS, used for cell culture, was included as background control and the isotype control and first antibody omission were used as negative controls. Proteins were visualized using Fluorochem HD2 chemiluminescencent workstation (Alpha Innotech) as described by the manufacturer.

\subsection{Immunofluorescence}

SwBMDCs were fixed with $4 \%$ paraformaldehyde, permeabilized in $0.5 \%$ Triton $\mathrm{X}-100$ and incubated with blocking solution ( $3 \%$ bovine serum albumin $(\mathrm{BSA}) / 1 \times \mathrm{PBS}$ ) for $1 \mathrm{~h}$ at RT. Labelling of PTX3 producing cells was performed using two different antibodies: rat anti-human PTX3 MNB4 and 16B5 (Camozzi et al., 2006). These primary antibodies (1:100 dilution) were incubated $\mathrm{ON}$ at $4{ }^{\circ} \mathrm{C}$. Detection of IV infected cells was determined using mouse anti-NP antibody (HB65, ATCC), incubated ON at $4{ }^{\circ} \mathrm{C}$; anti swine leucocyte antigen class II (SLAII-DR, clone 1F12) (INIA, Madrid) (Bullido et al., 1997) antibody for cell phenotyping was used for $1 \mathrm{~h}$ at RT.

For immunofluorescence, rat anti-HuPTX3 antibodies were detected using goat anti-rat IgG conjugated with Alexa 488 or Cy3 fluorophores (1:250 dilution) (Invitrogen). The rest of primary antibodies against cell markers and IV were detected using goat anti-mouse IgG secondary antibodies (1:250 dilution) coupled to Alexa 555 (Invitrogen) or Cy2 fluorophores (Jackson ImmunoResearch Europe Ltd.). Finally, nuclei were counterstained with DAPI and mounted in Fluoprep (BioMérieux, France). Negative control procedures were applied for all immunofluorescent tests. Specifically, in each specimen, negative controls to assess false positivity, autofluorescence and cross-reactivity were performed. Lack of the primary antibody was used as negative control.

\subsection{Microscopy and image analysis}

Fluorescent sections were viewed on a Nikon eclipse 90i epifluorescence microscope equipped with a DXM $1200 \mathrm{~F}$ camera (Nikon Corporation, Japan). To assess the colocalisation between different fluorescent labels, image stacks of sections were captured using the Leica TCS SP5 confocal microscope (x 63/NA 1.4 objective). Images were processed by using the LAS AF Lite programme from Leica and Image J v1.42k software [http://rsb.info.nih.gov/ij]. All sections were systematically examined in full and pictures were taken as representative areas for each section.

\subsection{Experimental infection designs}

SwPTX3 was determined in serum samples in pigs coming from an experimental infection with the new human pandemic swine influenza virus published recently (Busquets et al., 2010). Briefly, six pigs were intranasally infected with $3.5 \mathrm{ml}$ of $10^{6.7} \mathrm{TCID}_{50} / \mathrm{ml}$ of the new human pandemic A/Catalonia/63/2009 (H1N1) IV (A(H1N1)2009). Two pigs served as control animals, inoculated with $3.5 \mathrm{ml}$ of MEM. Pigs were clinically monitored daily and blood was drawn during the necropsy day ( 2 and 7 days post infection (p.i.)). Anti-influenza A virus NP antibody (IgG and IgA in serum) levels were measured and histopathological score of lung lesions was performed as previously described (Busquets et al., 2010). Thus, broncho-interstitial pneumonia intensity was assessed by means of a semiquantitative score ( $0-3$, indicating lack of, mild, moderate or severe pneumonia lesions, respectively).

Five pigs were intratracheally inoculated with $3 \mathrm{ml}$ of $10^{6.26} \mathrm{TCID}_{50} / \mathrm{ml}$ of H3N2 SwIV. Five pigs served as control animals, inoculated with $3 \mathrm{ml}$ of mock preparation. Pigs were clinically monitored daily and blood was drawn during the necropsy day (2 days post infection (p.i.)).

All experiments received prior approval from the Local Ethical Committee for Animal experimentation of the Institution (UAB). The treatment, housing and husbandry conditions conformed to the European Union Guidelines (The Council of the European Communities, 1986).

\subsection{ELISA assays}

SwPTX3 concentration in sera was determined as previously described (Salio et al., 2008) by sandwich ELISA against human PTX3 (HuPTX3) with the following antibodies: MNB4 and polyclonal rabbit antibody (Peri et al., 2000). Anti-influenza A virus nucleoprotein (NP) antibody levels were studied in serum using the ID Screen ${ }^{\circledR}$ Influenza A Antibody Competition ELISA (ID-Vet, Montpellier, France) following manufacturer's instructions. Detection of specific IgG and IgA in serum was performed modifying the ID Screen ${ }^{\circledR}$ Influenza A Antibody Competition ELISA with a goat anti-porcine IgG (Fc):HRP (Serotec AAI41P) antibody at $1: 100,000$ dilution or a goat antiporcine IgA:HRP (Serotec AAI40P) antibody at 1:10,000 dilution as secondary antibodies. Samples were used in triplicates and background values were subtracted from each sample.

\subsection{Statistical analysis}

All statistical analysis was performed using SPSS 15.0 software (SPSS Inc., Chicago, IL, USA). A $p$ value of less than 0.05 was considered significant with statistical tendencies reported when $0.05<p<0.1$. Descriptive statistics (means \pm SD) of PTX3 in different biological fluids are presented. The Shapiro Wilk's and the Levene test were used to evaluate the normality of the distribution of the examined quantitative variables and the homogeneity of variances, respectively. A Kruskal-Wallis test was used to study the association between PTX3 concentration in sera and day post-infection, the broncho-intersticial pneumonia intensity observed and the presence of polymorphonuclear (PMN) leucocyte (granulocytes) in the experimental infection with new human pandemic swine influenza virus. A regression analysis was carried out to estimate the association between 
PTX3 concentrations in sera and NP antibody titres (IgG and IgA) throughout this experimental infection study. A KruskalWallis test was also used to study the association between PTX3 concentration in sera and the subtype of swine influenza virus used in the other experimental infection. In all the cases, a non-parametric test (Mann-Whitney) was used to compare PTX3 between experimental different groups.

\section{Results}

\subsection{In silico comparison and Western blot analysis}

Results in Fig. 1A shows the alignment (Crustal W, European Bioinformatics Institute) of HuPTX3, murine PTX3 (MuPTX3) and SwPTX3. HuPTx3 and SwPTX3 proteins exhibited an aa identity of $84 \%$, as also shown in a recent study performed by Cabiati et al. (Cabiati et al., 2012). The alignment of HuPTX3 and SwPTX3 aa sequences showed that epitope (306-312) was fully conserved in both species (Fig. 1A), suggesting that 16B5 could be crossreactive for SwPTX3, whereas the epitope (87-99) was not entirely conserved in both species. Glutamic acid (E) at positions 90 and 97 were replaced by Alanine (A) and Glycine $(G)$ respectively (Fig. 1A). Thus, the cross-reactive activity of MNB4 might not be complete. Also, the in silico analysis used by running the NetNglyc server predicting
$\mathrm{N}$-glycosilation sites, showed that SwPTX3 lacks the NGlycosilation site present in human and murine PTX3 (Fig. 1A).

To gain further insight into the cross-reactivity of both monoclonal antibodies in porcine samples, SwPTX3 production in SwIV infected (Fig. 1B, lane 2), LPS (Fig. 1B, lane 3) and RHDV-VLP (Fig. 1B, lane 6) stimulated SwBMDCs were detected by Western blot using $16 \mathrm{~B} 5$ antibody as primary antibody. Myeloid dendritic cells (DCs) were selected because they are described as a major source of PTX3 (Deban et al., 2011; Inforzato et al., 2011). MNB4 was also used to detect SwPTX3 (data not shown). Lanes 2, 3 and 6 specifically showed a band compatible with the expected molecular mass of SwPTX3. The same results were obtained from both antibodies in Western blot, but 16B5 gave a stronger signal than MNB4 (data not shown), which was reasonable considering the sequence homology of each binding epitope (Fig. 1A). Basal level of SwPTX3 was also detected in unstimulated or uninfected cell lysate (data not shown) similarly as showed by other works (Doni et al., 2003; Jaillon et al., 2007).

Swine PTX3 shows an approximate molecular weight (MW) of $40 \mathrm{KDa}$ (Fig. 1B), while the human (Fig. 1B, lane 5) and murine PTX3 (Fig. 1B, lane 1) proteins were detected at an approximate MW of $45 \mathrm{KDa}$, as previously described by Bottazi et al. (Bottazzi et al., 1997) and

A

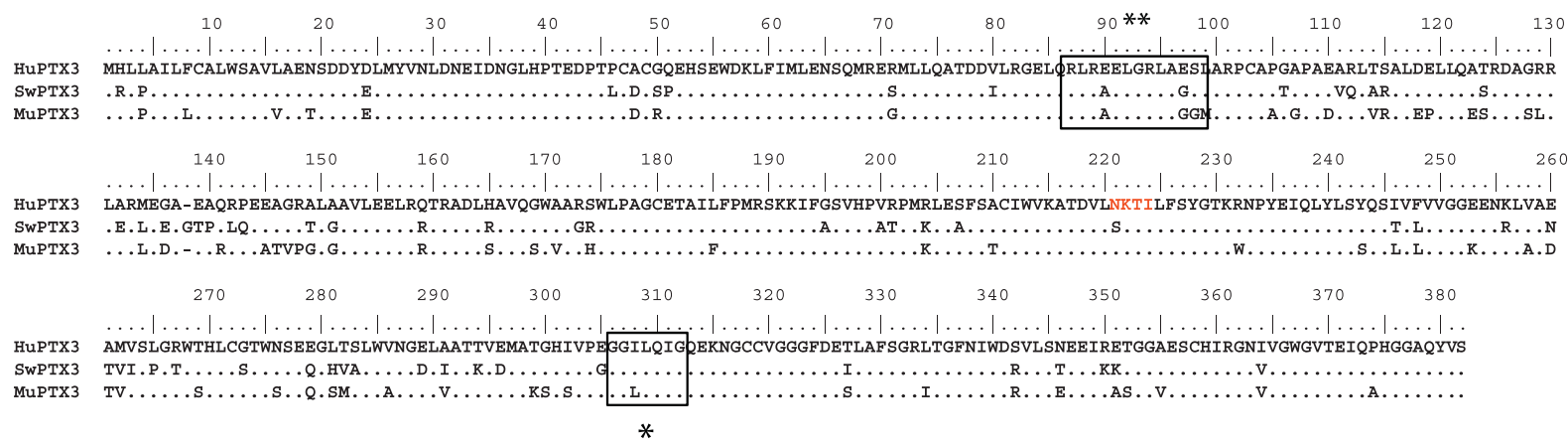

B

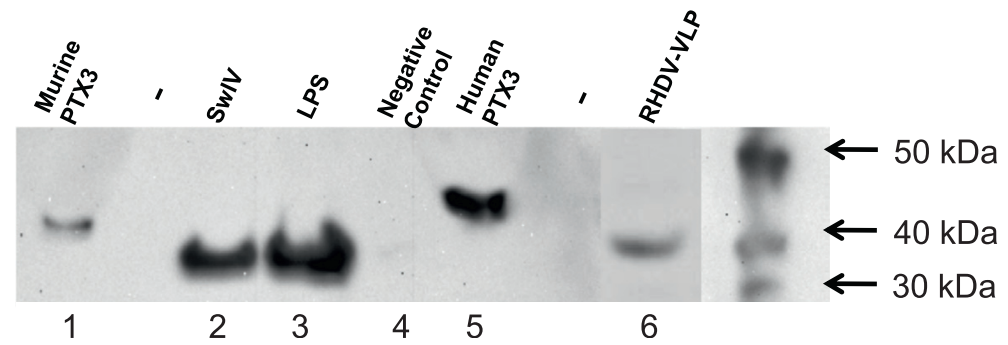

Fig. 1. Swine PTX3 characterization. (A) Alignment analysis of amino acid (aa) residues for human (HuPTX3), swine (SwPTX3) and murine PTX3 (MuPTX3). $\left({ }^{*}\right)$ Black squares indicated the 16B5 binding epitope in the $C$ terminal part, which is fully conserved in HuPTX3 and SwPTX3 sequences. (**) Black square indicate the MNB4 binding epitope in the N terminal part. This epitope is not totally conserved between HuPTX3 and SwPTX3 because Glutamic acid (E) at positions 90 and 97 is replaced by Alanine (A) and Glycine (G) respectively. Red NKTI aa indicates the Glycosilation sites in human PTX3. (B) Western blot with SDS-PAGE. Western blot using $16 \mathrm{~B} 5$ as primary antibody, revealed distinct masses for PTX3 protein corresponding to around $40 \mathrm{kDa}$ (lanes 2 , 3 and 6 ). No band was detected with the negative control (cell culture medium) (Negative Control, lane 4). MuPTX3 protein (lane 1) and HuPTX3 protein (lane 5) showed previously described molecular weight of approx. $45 \mathrm{kDa}$. Detection of SwPTX3 was also tested in samples from SwIV-infected (SwIV, lane 2 ), LPSstimulated (LPS, lane 3) and RHDV-VLP-stimulated (RHDV-VLP, lane 6) SwBMDC culture supernatant at overnight incubation. Representative results from three independent experiments. 
Scarchilli et al. (Scarchilli et al., 2007) respectively. This is due to the presence of $\mathrm{N}$-glycosylation site in the Cterminal domain (Bottazzi et al., 1997; Inforzato et al., 2006) of human and murine PTX3. The different mobility of SwPTX3 in the SDS gel compared with HuPTX3 and MuPTX3 might be due to the in silico differential predicted N-glycosilation (Fig. 1A).

\subsection{Immunofluorescence analysis of SwBMDCs}

SwBMDCs able to produce SwPTX3 were also identified by immunohistochemistry using MNB4 and 16B5 as primary antibodies (Fig. 2). A basal level of SwPTX3 staining was detected in the uninfected or unstimulated cells (Fig. 2A), which increased in SwBMDCs infected with SwIV (Fig. 2B). However SwPTX3 staining was observed not only in cells positive for NP as infected cells but also in cells negative for NP staining (Fig. 2B). Besides, PTX3 staining was detected in LPS-stimulated SwBMDCs (Fig. 2C) and also in RHDV-VLP-stimulated SwBMDCs (Fig. 2D). Therefore, these results suggested that SwPTX3 was expressed in SwBMDC.

\subsection{PTX3 expression in A(H1N1)2009 and SwIV (H3N2 and} H1N2) experimental infections

SwPTX3 concentration in serum increased at day 7 pi in A(H1N1)2009 infected animals (Table 1) but this difference was not significantly different throughout time $(p=0.15)$ probably due to the statistical potency of the assay and the variability observed between animals. However, SwPTX3 concentration in serum significantly correlated with specific $\operatorname{IgG}(r=0.71$ and $p=0.002)$ and $\operatorname{IgA}$ NP specific antibodies ( $r=0.84$ and $p=0.002)$ (Table 1$)$. On the other hand, lesion score (between 2 and 3 ) progressively increased after day 2 pi in infected animals but PTX3 concentration in serum did not show any association neither with lung lesion, measured through a lung histopathological lesion score (0-3) (Table 1) nor with the presence of polymorphonuclear (PMN) leucocyte (granulocytes) at day 7 pi (Table 1).

Additionally, serum SwPTX3 production in SwIV (H3N2 and $\mathrm{H} 1 \mathrm{~N} 2$ ) experimentally infected animals were studied. In these acute infections, PTX3 concentration in serum was significantly different between control and infected
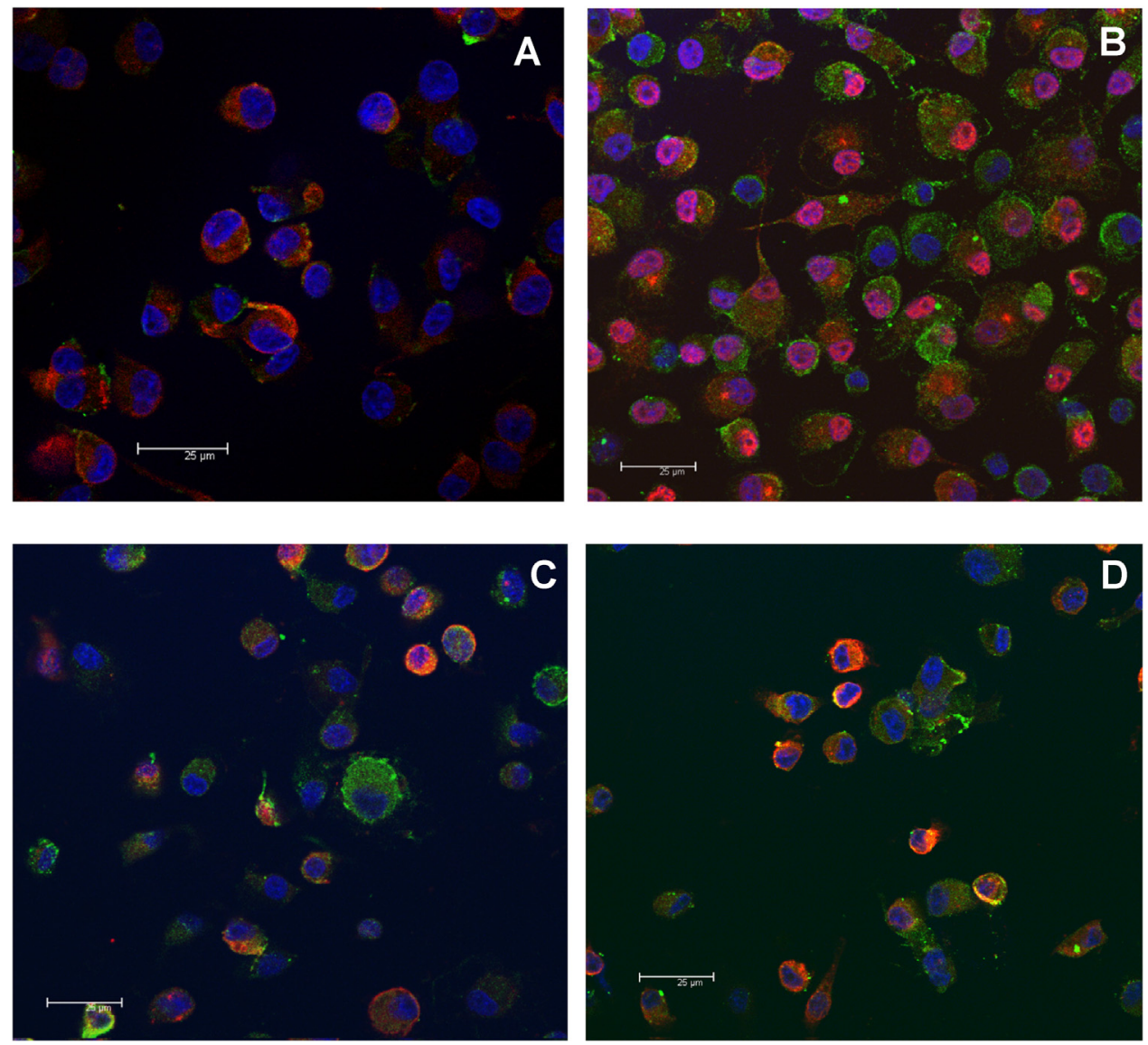

Fig. 2. PTX3 expression in SwBMDCs using anti human PTX3 MNB4 antibody. PTX3 immunostaining appears in green and nuclei in blue (DAPI). (A) Unstimulated or uninfected SwBMDCs (experiment control) at $8 \mathrm{~h}$. Red: SLAII positive cells. Bar $=25 \mu \mathrm{m}$. (B) SwIV infected SwBMDCs at $8 \mathrm{~h}$. Red: NP staining of influenza virus positive cells. Fuchsia: co-localization of blue (nucleus) and red (NP). Bar $=25 \mu \mathrm{m}$. (C) LPS-stimulated SwBMDCs at 8 h. Red: SLAII positive cells. Yellow: co-localization of green (PTX3) and red (SLAII). Bar $=25 \mu \mathrm{m}$. (D) RHDV-VLPs-stimulated SwBMDCs at 8 h. Red: SLAII positive cells. Yellow: co-localization of green (PTX3) and red (SLAII). Bar $=25 \mu \mathrm{m}$. Representative results from three independent experiments. (For interpretation of the references to colour in this figure legend, the reader is referred to the web version of this article.) 


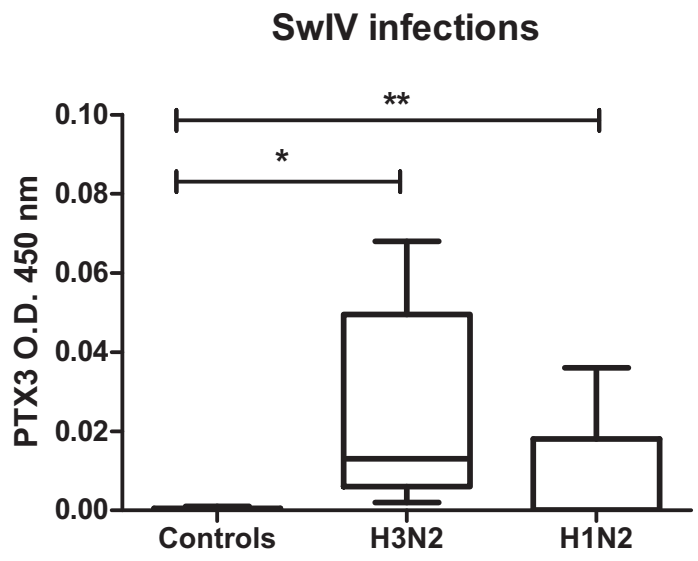

Fig. 3. PTX3 expression in serum during SwIV H3N2 and H1N2 experimental infection. Box-and-whisker plot is used to represent the data. Symbols ${ }^{*}$ and ${ }^{* *}$ indicate a statistical difference between means $(p<0.05)$.

animals $(p=0.02)$. Moreover, H3N2 infected pigs showed higher SwPTX3 serum levels compared with control $(p=0.01)$ or H1N2 infected animals (statistical tendency, $p=0.08)$ (Fig. 3).

\section{Discussion}

PTX3 is an important component of the humoral innate immunity and a study on the evolution of pentraxin family have shown that PTX3 originated directly from the common ancestors, very early in the evolution of pentraxins, at the divergence of vertebrates (Martinez de la Torre et al., 2010). An additional characterization of horse PTX3 (Ramery et al., 2010) and pig PTX3 mRNA (Cabiati et al., 2012) were recently published. In our study SwPTX3 was characterized using cross-reactive antibodies and the results further support the long pentraxin 3 homology between mammals.

Using anti HuPTX3 antibodies 16B5 and MNB4, recognizing respectively the $\mathrm{C}$-terminal and the $\mathrm{N}$-terminal domains, the molecular weight observed on Western blot under reducing condition is approximately $40 \mathrm{kDa}$. On the other hand, murine and human PTX3 proteins are seen as a $45 \mathrm{kDa}$ band (Bottazzi et al., 1997) due to the presence of a glycosilation site that is lacking in the SwPTX3. While these proteins share a high homology among the different species, it seems that they present a different pattern of post translational modifications. Why the SwPTX3 has lost a glycosilation site during evolution? Does it affect its function in the innate immune response? These questions will remain unanswered awaiting for future studies.

An important finding was the description for the first time that these antibodies with mapped epitopes against HuPTX3 cross-react with porcine samples, representing a good tool in SwPTX3 research, since nowadays there are no specie-specific antibodies against the porcine protein. MNB4 antibody has shown less affinity with porcine samples by Western blot (data not shown). Western blot and immunofluorescence determined that SwPTX3 protein is expressed in SwBMDCs, after infection with SwIV and also after stimulation with LPS and calicivirus-like particles (RHDV-VLPs) (Crisci et al., 2012). Uninfected immature SwBMDCs have shown a basal level of PTX3 in steady state condition detected by immunofluorescence (Fig. 2A) and Western blot (data not shown). This result is comparable with a previous study with human myeloid DCs (Doni et al., 2006) or with data by Jaillon et al. (Jaillon et al., 2007) who described the expression of PTX3 transcripts confined to human immature myeloid elements. Besides, variability due to different species and different culture systems cannot be ruled out.

Influenza virus directly stimulates SwPTX3 production in infected SwBMDCs but it seems to be not only a direct inducer. Indeed, SwBMDCs have shown positive labelling for SwPTX3 not only in cells infected with SwIV and therefore positive for NP protein, but also in uninfected cells negative for NP staining (Fig. 2). A plausible explanation would be that SwPTX3 expression may correlate with a different stage of IV infection. Alternatively, SwIV could activate secretion of soluble proteins (e.g. cytokines) in SwIV infected cells that may act as intermediate mediators for SwPTX3 expression in NP negative cells. Also, it could be due to different kinetics in SwPTX3 compared with NP protein in the cell.

To further investigate SwPTX3 induction after acute airway injury, an experimental infection in pigs using different IV subtypes was performed. SwPTX3 concentration correlate with viral NP specific antibodies (IgG and $\operatorname{IgA}$ ) in serum but not with the lesion lung score due to a A(H1N1)2009 IV infection. This degree of correlation between the specific IV humoral responses and the production of SwPTX3 in serum could be observed with the data from day 0 to 7 pi, before the normal development

Table 1

Summary of immune responses and lesion score in influenza A(H1N1)2009 experimental infection in pigs.

\begin{tabular}{|c|c|c|c|c|c|c|c|c|}
\hline \multirow[t]{2}{*}{ Animal } & \multicolumn{2}{|c|}{ Controls } & \multicolumn{2}{|l|}{ D2 } & \multicolumn{4}{|l|}{ D7 } \\
\hline & 1 & 2 & 3 & 4 & 7 & 8 & 9 & 10 \\
\hline $\begin{array}{l}\text { PTX3 serum O.D. } \\
\text { Serum }\end{array}$ & 0 & 0 & 0.03 & 0.02 & 0.11 & 0 & 0.04 & 0.06 \\
\hline NP-IgG & 0.0007 & 0 & 0.0152 & 0.0022 & 0.0327 & 0.0012 & 0.0017 & 0.0082 \\
\hline NP-IgA & 0.0107 & 0.0107 & 0.0072 & 0.0047 & 0.0387 & 0.0097 & 0.0187 & 0.0232 \\
\hline Lesion score & 0 & 0 & 2 & 1 & 2 & 3 & 3 & 3 \\
\hline PMN & No & No & No & No & Yes & No & No & Yes \\
\hline
\end{tabular}

PTX3 (O.D. at $450 \mathrm{~nm}$ ) and NP-specific IgG and IgA antibodies (O.D. at $450 \mathrm{~nm}$ ) in serum.

Lesion score (1-3) and presence of polymorphonuclear leucocyte (granulocytes) (PMN) in the lesions.

D2 and D7 indicate the days post infection. 
of anti-NP antibodies which occurs at day 10 pi (Lange et al., 2009). Additionally, SwPTX3 increased mainly after day 2, resembling results with other acute phase proteins (APPs) (Brookes et al., 2010). During SwIV, H3N2 subtype induced higher levels of SwPTX3 in serum compared to controls and H1N2 infected animals. This difference could be related with different infection kinetics, confirmed also by other IV studies on acute phase proteins in pigs (Barbe et al., 2011; Brookes et al., 2010; Pomorska-Mol et al., 2012a,b). However, possible differences in sensitivity of cross reactive human PTX3 antibodies in porcine samples have to be taken into account.

This is the first preliminary characterization of SwPTX3 in the porcine innate immune system. PTX3 has been correlated with outcomes of pathological conditions in several species and these results will encourage ongoing research efforts as candidate novel marker for acute inflammatory and infectious pathology in pigs.

\section{Conflict of interest}

The authors declare that they have no conflict of interests.

\section{Acknowledgements}

We thank Bárcena J. for calicivirus-like particles (RHDVVLPs); Domínguez J. for the antibodies and swine molecules; Usero L. and Roura C. for anti-rat and antimouse fluorescent antibodies; Ballester M., Cordoba L., Mussá T., Martinez-Orellana P., for the technical assistance; Baratelli M. for PTX3 amino acid analysis; Bertho, N. and Maisonnasse, P. for scientific support. We thank Alicia Solórzano for critically reviewing the manuscript.

This work was partly funded by the projects AGL200912945-C02-01 and AGL2010-22200-C02-01 by the Spanish Government and FP-7-2008-1, 228394, NADIR project funded by the EU. The contribution of Regione Lombardia (project Metadistretti-SEPSIS), the European Research Council (project HIIS) and the Fondazione Humanitas per la Ricerca is gratefully acknowledged.

\section{References}

Barbe, F., Atanasova, K., Van Reeth, K., 2011. Cytokines and acute phase proteins associated with acute swine influenza infection in pigs. Vet. J. 187, 48-53.

Bottazzi, B., Doni, A., Garlanda, C., Mantovani, A., 2010. An integrated view of humoral innate immunity: pentraxins as a paradigm. Annu. Rev. Immunol. 28, 157-183.

Bottazzi, B., Garlanda, C., Cotena, A., Moalli, F., Jaillon, S., Deban, L., Mantovani, A., 2009. The long pentraxin PTX3 as a prototypic humoral pattern recognition receptor: interplay with cellular innate immunity. Immunol. Rev. 227, 9-18.

Bottazzi, B., Vouret-Craviari, V., Bastone, A., De Gioia, L., Matteucci, C., Peri, G., Spreafico, F., Pausa, M., D’Ettorre, C., Gianazza, E., Tagliabue, A., Salmona, M., Tedesco, F., Introna, M., Mantovani, A., 1997. Multimer formation and ligand recognition by the long pentraxin PTX3. Similarities and differences with the short pentraxins C-reactive protein and serum amyloid P component. J. Biol. Chem. 272, 3281732823.

Brookes, S.M., Nunez, A., Choudhury, B., Matrosovich, M., Essen, S.C., Clifford, D., Slomka, M.J., Kuntz-Simon, G., Garcon, F., Nash, B., Hanna, A., Heegaard, P.M., Queguiner, S., Chiapponi, C., Bublot, M., Garcia, J.M., Gardner, R., Foni, E., Loeffen, W., Larsen, L., Van Reeth, K., Banks, J., Irvine, R.M., Brown, I.H., 2010. Replication, pathogenesis and transmission of pandemic (H1N1) 2009 virus in non-immune pigs. PLoS One 5, e9068.

Bullido, R., Domenech, N., Alvarez, B., Alonso, F., Babin, M., Ezquerra, A. Ortuno, E., Dominguez, J., 1997. Characterization of five monoclonal antibodies specific for swine class II major histocompatibility antigens and crossreactivity studies with leukocytes of domestic animals. Dev. Comp. Immunol. 21, 311-322.

Busquets, N., Segales, J., Cordoba, L., Mussa, T., Crisci, E., Martin-Valls, G.E., Simon-Grife, M., Perez-Simo, M., Perez-Maillo, M., Nunez, J.I., Abad, F.X., Fraile, L., Pina, S., Majo, N., Bensaid, A., Domingo, M., Montoya, M., 2010. Experimental infection with H1N1 European swine influenza virus protects pigs from an infection with the 2009 pandemic H1N1 human influenza virus. Vet. Res. 41, 74.

Cabiati, M., Caselli, C., Savelli, S., Prescimone, T., Lionetti, V., Giannessi, D. Del Ry, S., 2012. Exploring PTX3 expression in Sus scrofa cardiac tissue using RNA sequencing. Regul. Pept. 174, 1-5.

Camozzi, M., Rusnati, M., Bugatti, A., Bottazzi, B., Mantovani, A., Bastone, A., Inforzato, A., Vincenti, S., Bracci, L., Mastroianni, D., Presta, M., 2006. Identification of an antiangiogenic FGF2-binding site in the $\mathrm{N}$ terminus of the soluble pattern recognition receptor PTX3. J. Biol. Chem. 281, 22605-22613.

Cieslik, P., Hrycek, A., 2012. Long pentraxin 3 (PTX3) in the light of its structure, mechanism of action and clinical implications. Autoimmunity 45, 119-128.

Crisci, E., Fraile, L., Moreno, N., Blanco, E., Cabezon, R., Costa, C., Mussa, T., Baratelli, M., Martinez-Orellana, P., Ganges, L., Martinez, J., Barcena, J., Montoya, M., 2012. Chimeric calicivirus-like particles elicit specific immune responses in pigs. Vaccine 30, 2427-2439.

Deban, L., Jaillon, S., Garlanda, C., Bottazzi, B., Mantovani, A., 2011. Pentraxins in innate immunity: lessons from PTX3. Cell Tissue Res. 343, 237-249

Doni, A., Michela, M., Bottazzi, B., Peri, G., Valentino, S., Polentarutti, N., Garlanda, C., Mantovani, A., 2006. Regulation of PTX3, a key component of humoral innate immunity in human dendritic cells: stimulation by IL-10 and inhibition by IFN-gamma. J. Leukoc. Biol. 79, 797802 .

Doni, A., Peri, G., Chieppa, M., Allavena, P., Pasqualini, F., Vago, L., Romani, L., Garlanda, C., Mantovani, A., 2003. Production of the soluble pattern recognition receptor PTX3 by myeloid, but not plasmacytoid, dendritic cells. Eur. J. Immunol. 33, 2886-2893.

He, X., Han, B., Liu, M., 2007. Long pentraxin 3 in pulmonary infection and acute lung injury. Am. J. Physiol. Lung Cell. Mol. Physiol. 292, L1039L1049.

Higgins, D.G., Thompson, J.D., Gibson, T.J., 1996. Using CLUSTAL for multiple sequence alignments. Methods Enzymol. 266, 383-402.

Inforzato, A., Jaillon, S., Moalli, F., Barbati, E., Bonavita, E., Bottazzi, B., Mantovani, A., Garlanda, C., 2011. The long pentraxin PTX3 at the crossroads between innate immunity and tissue remodelling. Tissue Antigens 77, 271-282.

Inforzato, A., Peri, G., Doni, A., Garlanda, C., Mantovani, A., Bastone, A., Carpentieri, A., Amoresano, A., Pucci, P., Roos, A., Daha, M.R., Vincenti, S., Gallo, G., Carminati, P., De Santis, R., Salvatori, G., 2006. Structure and function of the long pentraxin PTX3 glycosidic moiety: finetuning of the interaction with $\mathrm{C} 1 \mathrm{q}$ and complement activation. Biochemistry 45, 11540-11551.

Jaillon, S., Peri, G., Delneste, Y., Fremaux, I., Doni, A., Moalli, F., Garlanda, C., Romani, L., Gascan, H., Bellocchio, S., Bozza, S., Cassatella, M.A., Jeannin, P., Mantovani, A., 2007. The humoral pattern recognition receptor PTX3 is stored in neutrophil granules and localizes in extracellular traps. J. Exp. Med. 204, 793-804.

Job, E.R., Deng, Y.M., Tate, M.D., Bottazzi, B., Crouch, E.C., Dean, M.M., Mantovani, A., Brooks, A.G., Reading, P.C., 2010. Pandemic H1N1 influenza A viruses are resistant to the antiviral activities of innate immune proteins of the collectin and pentraxin superfamilies. J. Immunol. 185, 4284-4291.

Lange, E., Kalthoff, D., Blohm, U., Teifke, J.P., Breithaupt, A., Maresch, C., Starick, E., Fereidouni, S., Hoffmann, B., Mettenleiter, T.C., Beer, M., Vahlenkamp, T.W., 2009. Pathogenesis and transmission of the novel swine-origin influenza virus A/H1N1 after experimental infection of pigs. J. Gen. Virol. 90, 2119-2123.

Martinez de la Torre, Y., Fabbri, M., Jaillon, S., Bastone, A., Nebuloni, M., Vecchi, A., Mantovani, A., Garlanda, C., 2010. Evolution of the pentraxin family: the new entry PTX4. J. Immunol. 184, 5055-5064.

Mussa, T., Rodriguez-Carino, C., Pujol, M., Cordoba, L., Busquets, N., Crisci, E., Dominguez, J., Fraile, L., Montoya, M., 2011. Interaction of porcine conventional dendritic cells with swine influenza virus. Virology 420, 125-134.

Peri, G., Introna, M., Corradi, D., Iacuitti, G., Signorini, S., Avanzini, F., Pizzetti, F., Maggioni, A.P., Moccetti, T., Metra, M., Cas, L.D., Ghezzi, P., Sipe, J.D., Re, G., Olivetti, G., Mantovani, A., Latini, R., 2000. PTX3, A 
prototypical long pentraxin, is an early indicator of acute myocardial infarction in humans. Circulation 102, 636-641.

Pomorska-Mol, M., Markowska-Daniel, I., Kwit, K., 2012a. Immune and acute phase response in pigs experimentally infected with H1N2 swine influenza virus. FEMS Immunol. Med. Microbiol. 66, 334-342.

Pomorska-Mol, M., Markowska-Daniel, I., Pejsak, Z., 2012b. Acute phase protein response during subclinical infection of pigs with H1N1 swine influenza virus. Vet. Microbiol. 159, 499-503.

Ramery, E., Fievez, L., Fraipont, A., Bureau, F., Lekeux, P., 2010. Characterization of pentraxin 3 in the horse and its expression in airways. Vet Res. 41, 18.

Reading, P.C., Bozza, S., Gilbertson, B., Tate, M., Moretti, S., Job, E.R., Crouch, E.C., Brooks, A.G., Brown, L.E., Bottazzi, B., Romani, L.,
Mantovani, A., 2008. Antiviral activity of the long chain pentraxin PTX3 against influenza viruses. J. Immunol. 180, 3391-3398.

Reed, L.J., Muench, H., 1938. A simple method of estimating fifty per cent endpoint. Am. J. Hyg. 27, 493-497.

Salio, M., Chimenti, S., De Angelis, N., Molla, F., Maina, V., Nebuloni, M. Pasqualini, F., Latini, R., Garlanda, C., Mantovani, A., 2008. Cardioprotective function of the long pentraxin PTX3 in acute myocardial infarction. Circulation 117, 1055-1064.

Scarchilli, L., Camaioni, A., Bottazzi, B., Negri, V., Doni, A., Deban, L., Bastone, A., Salvatori, G., Mantovani, A., Siracusa, G., Salustri, A., 2007. PTX3 interacts with inter-alpha-trypsin inhibitor: implications for hyaluronan organization and cumulus oophorus expansion. J. Biol. Chem. 282, 30161-30170. 